\title{
miR-218 suppresses gastric cancer cell proliferation and invasion via regulation of angiopoietin-2
}

\author{
SIFENG TANG $^{1,2^{*}}$, DEYOU WANG ${ }^{2 *}$, QIWEN ZHANG ${ }^{2}$ and LEPING LI ${ }^{1,3}$ \\ ${ }^{1}$ Department of Gastrointestinal Surgery, School of Medicine, Shandong University, Jinan, Shandong 250012; \\ ${ }^{2}$ Department of General Surgery, Laiwu People's Hospital, Laiwu, Shandong 271100; ${ }^{3}$ Department of General Surgery, \\ Shandong Provincial Hospital Affiliated to Shandong University, Jinan, Shandong 250021, P.R. China
}

Received December 5, 2015; Accepted September 1, 2016

DOI: $10.3892 /$ etm.2016.3893

\begin{abstract}
Novel targeted therapies need to be developed for gastric cancer, the third most common cancer type and the second most common cause of cancer-related mortality in China. Previous studies indicate that angiopoietin (Ang)-2 serves a role in the proliferation, migration, invasion and adhesion of malignant cells. The present study identified, using functional studies, that exogenous expression of miR-218 increased migration of NCI-87 and HGC-27 gastric cancer cells, which coincided with a reduction in the expression of Ang-2. In addition, intratumoral delivery of miR-218 inhibited proliferation and angiogenesis of gastric cancer cells in vivo, with a corresponding decreased in Ang-2 expression. These results indicate that miR-218 serves an important role in gastric cancer tumorigenesis through regulating the expression of Ang-2. Therefore, components of miR-218/Ang-2 signaling could provide novel therapeutic targets for the treatment of gastric cancer.
\end{abstract}

\section{Introduction}

Gastric cancer is the second most common cause of cancer-related mortality in China (1). The highest gastric cancer mortality rates are in East Asia, particularly in Japan, China and Korea $(2,3)$. Numerous previous studies have demonstrated that angiogenesis serves a crucial role in the growth and metastasis of gastric cancer cells $(4,5)$.

Correspondence to: Dr Qiwen Zhang, Department of General Surgery, Laiwu People's Hospital, 1 Wenhuabei Road, Laiwu, Shandong 271100, P.R. China

E-mail: dsyu2008@163.com

Professor Leping Li, Department of Gastrointestinal Surgery, School of Medicine, Shandong University, 44 Wehua Xi Road, Jinan, Shandong 250012, P.R. China

E-mail: lepng_li@163.com

*Contributed equally

Key words: miR-218, angiopoietin-2, gastric cancer, angiogenesis, proliferation
Angiopoietin (Ang)-2, a natural antagonist of the angiogenic Tie-2 receptor, and vascular endothelial growth factor are thought to be critical regulators of tumor angiogenesis (6). Accumulating evidence demonstrates that Ang-2 serves dual roles in tumorigenesis through promoting angiogenesis and directly stimulating cancer cell proliferation (7).

Angiogenesis is defined as the sprouting of blood vessels from pre-existing vessels. These new vessels can migrate towards the tumor, forming a new vascular network that enables a wide range of activities, such as cellular proliferation, migration, invasion, adhesion and formation of tubular structures (8). Four members of the Ang family have been identified, Ang-1, $-2,-3$ and -4 , which serve different roles in angiogenesis. Ang-1 is the primary agonist of the endothelial cell-specific receptor tyrosine kinase 2 (Tie2), inducing receptor autophosphorylation upon binding (9). Conversely, Ang-2 is an antagonist of Ang-1 that inhibits the autophosphorylation of Tie2, leading to vessel destabilization (9). However, the expression and role of Ang-2 in human gastric cancer remains unclear.

Micro RNAs (miRNAs) are a class of small noncoding RNAs that regulate post-transcriptional gene expression, through cleaving or inhibiting the translocation of mRNA molecules. A previous study demonstrated that miRNAs regulate angiogenesis by altering the response of endothelial cells to anti- or pro-angiogenic factors (10). miRNAs can promote or inhibit angiogenesis, for example, it has been reported that miR-126, miR-130a, miR-210 and miR-296 promote angiogenesis, whereas miR-221 and miR-222 inhibit angiogenesis (11). Although numerous miRNAs have been identified as oncogenes and tumor suppressors (12), to the best of our knowledge, in gastric cancer, these functions have not been associated with the targeting of Ang transcripts.

In the present study, miR-218 expression was markedly downregulated in NCI-87 gastric cancer cells, which exhibited increased invasion. In addition, exogenous expression of miR-218 in NCI-87 and HGC-27 gastric cancer cells increased migration, which is coincided with a reduction in the expression of Ang-2. Furthermore, intratumoral delivery of miR-218 inhibited gastric cancer cell proliferation and angiogenesis in vivo, accompanied with decreased Ang-2 expression. These results suggest that miR-218 suppresses gastric tumor growth and metastasis through regulation of Ang-2. 


\section{Materials and methods}

Cell culture. Human gastric cancer cell lines (MGC80-3, HGC-27 and NCI-N87) were obtained from American Type Culture Collection (Manassas, VA, USA) and cultured in RPMI-1640 media (Gibco; Thermo Fisher Scientific, Inc., Waltham, MA, USA), supplemented with $10 \%$ fetal bovine serum (FBS; Hyclone; GE Healthcare Life Sciences, Logan, UT, USA) $100 \mathrm{U} / \mathrm{ml}$ penicillin and $100 \mathrm{U} / \mathrm{ml}$ streptomycin (both Gibco; Thermo Fisher Scientific, Inc.) at $37^{\circ} \mathrm{C}$ in a humidified incubator containing $5 \% \mathrm{CO}_{2}$.

Cells were seeded into six-well plates and transfected when they were between 70 and $80 \%$ confluent. NCI- 87 and HGC-27 cells were transfected with 30 pmol pre-miR-218 (AM17100) or scrambled miRNA (negative control; AM17110) (both Ambion; Thermo Fisher Scientific, Inc.) using Lipofectamine RNAiMAX Reagent (Invitrogen; Thermo Fisher Scientific, Inc.) in Opti-MEM media (Gibco; Thermo Fisher Scientific, Inc.), according to the manufacturer's instructions. Total RNA and protein samples were prepared (as described below) $48 \mathrm{~h}$ following transfection.

In vitro invasion assay. The invasive ability of gastric cancer cell lines was examined using a modified two-chamber Transwell migration assay, as described previously (13). Briefly, $2 \times 10^{5}$ cells were untransfected or transfected with scramble or pre-miR-218 and seeded onto the upper side of 24-well Transwell plates (Corning Inc., Corning, NY, USA) coated with Matrigel (BD Biosciences, Heidelberg, Germany) diluted 1:2 with standard culture medium. The lower chamber was filled with culture media, as previously described. Following incubation at $37^{\circ} \mathrm{C}$ for $24 \mathrm{~h}$, cells on the upper side of the membrane were wiped away and the membrane was fixed with $4 \%$ paraformaldehyde and $0.25 \%$ glutaraldehyde. Cells on the lower side of the membrane were counted following staining with $0.5 \%$ methylene blue in $50 \%$ methanol. All invasion assay were performed in triplicate.

Reverse transcription-quantitative polymerase chain reaction (RT-qPCR) analysis. Total RNA was extracted from transfected or un-trasfected MGC80-3, HGC-27 and NCI-N87 cells using TRIzol reagent (Invitrogen; Thermo Fisher Scientific, Inc.) and treated with DNase I (Promega Corporation, Madison, WI, USA). RT was performed using the Moloney murine leukemia virus reverse transcriptase (M3681; Promega Corporation) and oligo(dT) 15 primers (C110B-C; Promega Corporation), according to the manufacturer's protocol. cDNA obtained was amplified through conventional PCR. Then, qPCR was performed using a StepOne Real-Time PCR system (Applied Biosystems; Thermo Fisher Scientific, Inc.), where DNA was detected with SYBR Green (K0252; Thermo Fisher Scientific, Inc.). miR-218 and Ang-2 expression levels were normalized against GAPDH and U6, and then measured using the comparative cycle threshold method (14). The following primers were used for qPCR: Ang-2 forward (F), 5'-AGATTT TGGACCAGACCAGTGA-3' and reverse (R), 5'-GGATGA TGTGCTTGTCTTCCAT-3'; GAPDH F, 5'-TGTGGGCAT CAATGGATTTGG-3' and R, 5'-ACACCATGTATTCCG GGTCAAT-3'; miR-218 F, 5'-AAGGGAGTCCAGTTTTCC CAGGAATCC-3' and R, 5'-GTCGTATCCAGTGCAGGG
TCCGAGGTATTCGCACTGGATACGAC-3'; and U6 F, 5'-CTCGCTTCGGCAGCACA-3', and R, 5'-AACGCTTCA CGAATTTGCGT-3'. qPCR was performed with the following cycles: $95^{\circ} \mathrm{C}$ for $10 \mathrm{~min}$, followed by $95^{\circ} \mathrm{C}$ for $15 \mathrm{sec}, 60^{\circ} \mathrm{C}$ for $30 \mathrm{sec}$, and $72^{\circ} \mathrm{C}$ for $30 \mathrm{sec}$ for 40 cycles.

Western blot analysis. To obtain protein, cells were washed with PBS and lysed in radioimmunoprecipitation assay lysis buffer, supplemented with a protease inhibitor cocktail (Roche Applied Science, Mannheim, Germany). Total protein was quantified using a bicinchoninic acid protein assay kit (P0009; Beyotime Institute of Biotechnology, Jiangsu, China). A total of $30 \mu \mathrm{g}$ total lysates were resolved using 10\% SDS-PAGE and transferred to a polyvinylidene difluoride membrane (Merck Millipore, Eschborn, Germany). The membranes were blocked in 5\% skimmed milk in Tris-buffered saline and Tween 20 for $1 \mathrm{~h}$, followed by incubation with primary anti-Ang-2 (dilution, 1:1,000; polyclonal; rabbit; ab8452) and anti-actin (dilution, 1:1,000; monoclonal; mouse; ab3280) antibodies overnight at $4^{\circ} \mathrm{C}$ (both Abcam, Cambridge, MA, USA). Then, the membranes were washed three times (5 min each) in Tris-buffered saline and Tween 20, and incubated with horseradish peroxidase-conjugated secondary antibody at room temperature for $1 \mathrm{~h}$ (polyclonal; goat anti-rabbit; 111-005-003; Jackson ImmunoResearch Laboratories, Inc., West Grove, PA, USA). Following washing, the bands were detected using an enhanced chemiluminescence reagent (Merck Millipore). The intensities of the resulting bands were quantified using the ChemiDoc MP system (Bio-Rad Laboratories, Inc., Hercules, CA, USA) and normalized against $\beta$-actin.

Ang-2 enzyme-linked immunosorbent assay (ELISA). Centriugation $(13,400 \times \mathrm{g})$ was performed at $4^{\circ} \mathrm{C}$ for $10 \mathrm{~min}$ $48 \mathrm{~h}$ post-transfection with pre-miR-218 or scrambled miRNA to remove particulates, and the concentration of Ang-2 in MGC80-3, HGC-27 and NCI-N87 cell culture supernatants was determined. Measurements were made using the Human Ang-2 Quantikine ELISA kit (DANG20; R\&D Systems; Minneapolis, MN, USA), according to the manufacturer's instructions.

Soft agar colony formation assay. Colony formation in soft agar was examined by plating $2.5 \times 10^{4}$ transfected cells in $0.4 \mathrm{ml}$ RPMI-1640 (supplemented with 100 units $/ \mathrm{ml}$ penicillin, $100 \mu \mathrm{g} / \mathrm{ml}$ streptomycin, $100 \mu \mathrm{g} / \mathrm{ml}$ amphotericin B, 3\% FBS and $0.3 \%$ low melting temperature SeaPlaque agarose) into 12-well plates (6 wells each for scrambled miRNA and pre-miR-218 transfected cells) coated with $0.8 \mathrm{ml}$ of $0.6 \%$ low melting temperature agarose. Platelet-derived growth factor-BB (50 ng/ml; 220-BB-010; R\&D Systems) was added to half of the wells of each type of transfected cells. Then, plates were incubated at $37^{\circ} \mathrm{C}$ for five days, over which colony formation, including colony number and size, was monitored.

In vivo tumor growth. A total of 24 male athymic immunocompromised BALB/c nude mice (age, 4-6 weeks; weight, 18-20 g) were purchased from the Model Animal Research Center of Nanjing University (Nanjing, China). Untransfected NCI-87 cells $\left[5 \times 10^{6}\right.$ resuspended in $100 \mu 1$ cold phosphate-buffered saline (PBS)] were subcutaneously injected into the right flank 
A

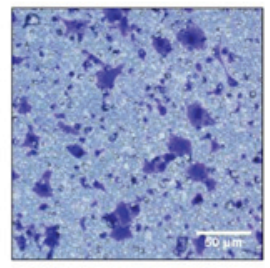

MGC80-3

B

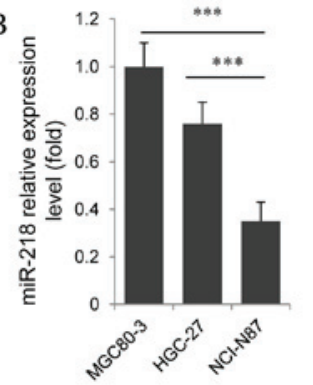

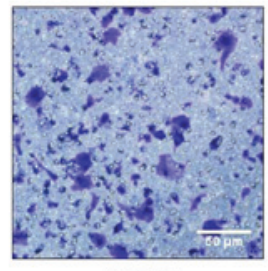

HGC-27

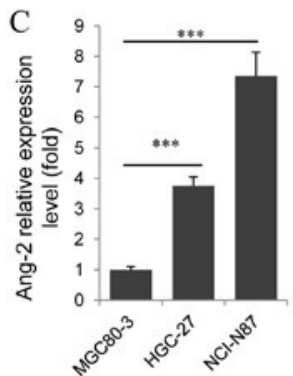

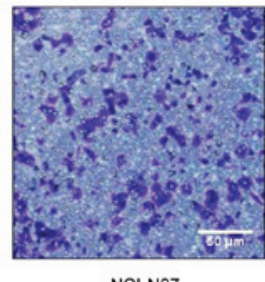

NCI-N87

D

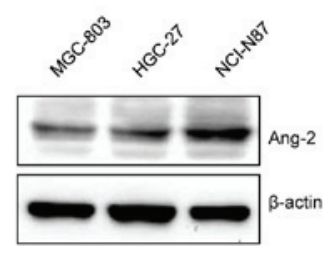

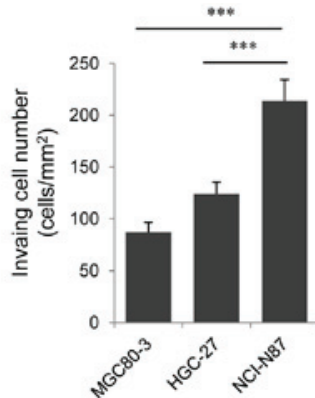

$\mathrm{E}$

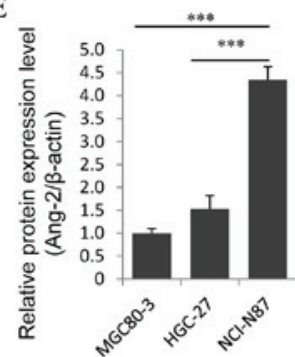

Figure 1. Decreased miR-218 expression is associated with increased Ang-2 expression and invasive ability in gastric cancer cell lines. (A) Representative images of the in vitro invasion assay (magnification, $\mathrm{x} 200$ ) stained with methylene blue and a graphical representation of each cell line. Scale bar, $50 \mu \mathrm{m}$. Reverse transcription-quantitative polymerase chain reaction analysis of the relative expression levels of (B) miR-218 normalized to U6 and (C) Ang-2 mRNA normalized to GAPDH, in gastric cancer cells. (D) Western blot of the protein expression levels of Ang-2 in gastric cancer cells. (E) Ang-2 protein levels determined by western blot image analysis. Results are presented as the mean \pm standard error of the mean of three independent experiments. ${ }^{* * *} \mathrm{P}<0.001$. miR, miRNA; Ang-2, angiopoietin-2.

of the mice. Tumor volume was measured every five days, as described previously (15). At 10 days following subcutaneous injection with NCI-87, mice were intratumorally injected with scrambled miRNA or pre-miR-218 for four weeks, twice a week. For each injection, $5 \mu \mathrm{g}$ miRNA was diluted in $50 \mu \mathrm{l}$ PBS. Following the study, mice were sacrificed via administraion of $5 \%$ chloral hydrate anesthesia $(0.1 \mathrm{ml} / 10 \mathrm{~g}$; Sinopharm Chemical Reagent Co., Ltd., Shanghai, China) and tumors dissected for western blotting (above) and immunohistochemistry (below). All animal studies were approved by the Jiangsu Cancer Hospital and Research Institute Animal Care and Use Committee (Jiangsu, China; approval no. 21040718) and carried out in accordance with the committee's Guide for the Care and Use of Laboratory Animals. Mice were kept in a temperature controlled room at $24 \pm 1{ }^{\circ} \mathrm{C}$ (40-60\% humidity) with a 12-h light/dark cycle, and ad libitum access to food and water.

Immunohistochemistry. Immunohistochemical analysis of tumor vascularization was performed using the analySIS system (Leica Microsystems GmbH, Wetzlar, Germany) Briefly, 5- $\mu \mathrm{m}$ formaldehyde-fixed, paraffin-embedded tumor sections were cut, mounted on slides coated with 3-(triethoxysilyl) propylamine (Sigma-Aldrich; Merck Millipore) and fixed at $37^{\circ} \mathrm{C}$ overnight. Following deparaffinization in xylene and rehydration with a series of graded alcohols, the slides were incubated in $\mathrm{H}_{2} \mathrm{O}_{2}$ to block endogenous peroxidase activity. Then, sections were incubated overnight at $4^{\circ} \mathrm{C}$ with anti-cluster of differentiation (CD) 31 (dilution, 1:200; 3528; Cell Signaling Technology, Inc., Danvers, MA, USA) or anti-marker of proliferation Ki-67 (dilution, 1:200; ab15580; Abcam) primary monoclonal antibodies. Following washing, the sections were incubated at room temperature with biotinylated goat anti-rabbit antibody (dilution, 1:300; BP-9100; Vector Laboratories, Inc., Burlingame, California, USA) for $1 \mathrm{~h}$. Then, sections were washed, treated with 3,3'-diaminobenzidine tetrahydrochloride and peroxidase activity visualized. Hematoxylin was used as a counterstain.

Vacularization evaluation. The amount of vascularization [microvessel density (MVD)] was calculated as the average number of Factor VIII-positive microvessels. Briefly, CD31-stained (3528; Cell Signaling Technology, Inc., Danvers, MA, USA) sections were scanned at a low power (magnification, $\mathrm{x} 100$ ) and areas with the highest quantity of microvessels were selected. Subsequently, microvessel counting was performed using an Asperio VERSA scanner (Leica Microsystems $\mathrm{GmbH})$ at $\mathrm{x} 200$ magnification in three different areas with the highest quantity of microvessel observed at x100 magnification, and the mean value was taken as the MVD for further analysis. Any clearly stained endothelial cells or cell clusters were considered to be a single microvessel. Lumens and large vessels were automatically excluded from the analysis.

Statistical analysis. Results are expressed as the mean \pm the standard error of the mean from $\geq 3$ independent experiments. Statistical analysis of a normal distribution of data was performed using a two-tailed Student's t-test using SPSS 10.0 (SPSS, Inc., Chicago, IL, USA) with post-hoc tests. $\mathrm{P}<0.05$ and $\mathrm{P}<0.01$ were considered to indicate significant and highly significant differences, respectively.

\section{Results}

Invasive gastric cancer cell lines have increased miR-218 and decreased Ang-2 mRNA expression levels. To investigate 

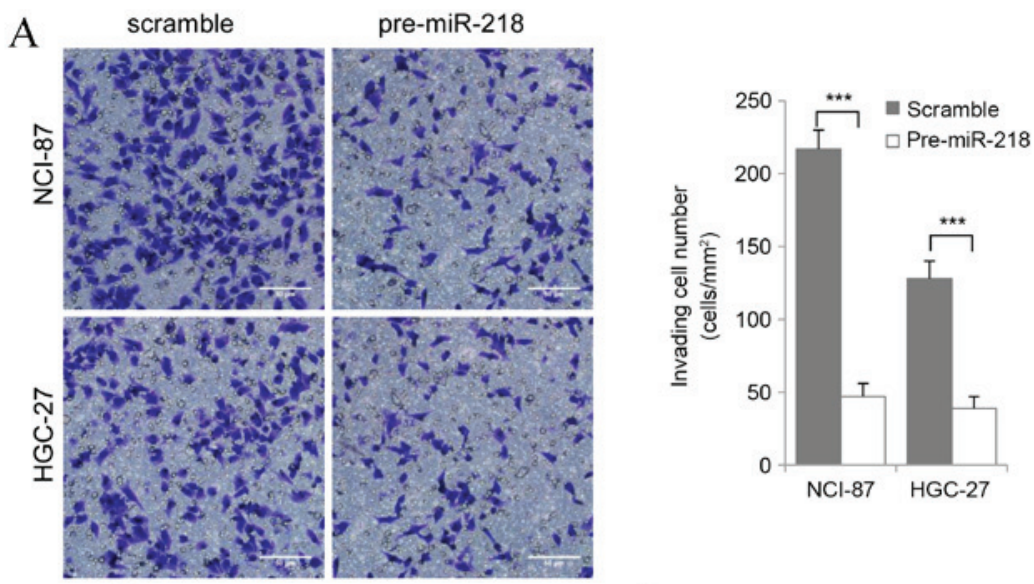

B

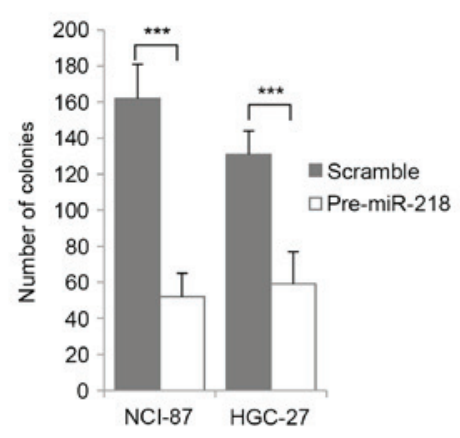

C

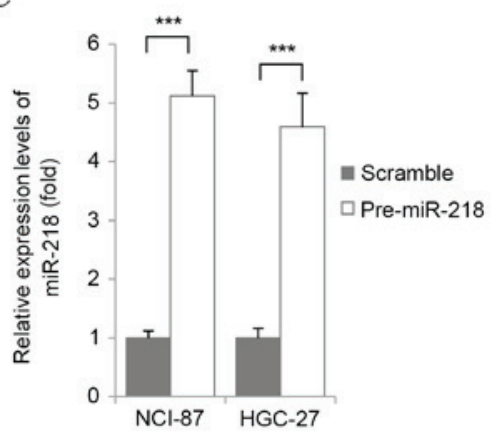

$\mathrm{D}$

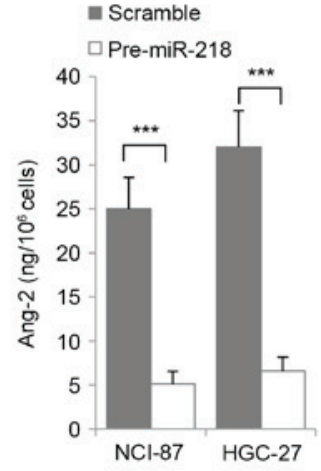

$\mathrm{E}$

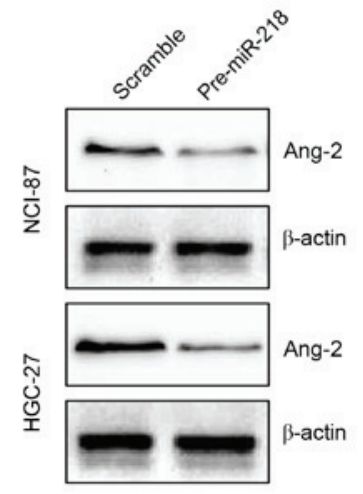

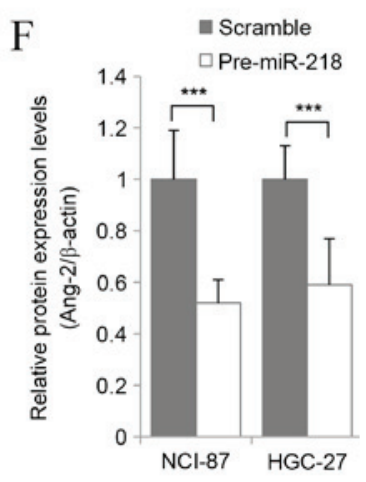

Figure 2. miR-218 inhibits the proliferation and migration of gastric cancer cells via regulating the expression of Ang-2. (A) Representative images (magnification, x200) and quantification of the in vitro invasion assay for NCI-87 and HGC-27 cells transfected with pre-miR-218 or scramble using Transwell assay. Scale bar, $50 \mu \mathrm{m}$. (B) Results of the soft agar colony formation assay. (C) Reverse transcription-quantitative polymerase chain reaction analysis of miR-218 expression levels in transfected cells. (D) ELISA detection of Ang-2 protein levels in the culture supernatant. (E) Western blot of the protein expression levels of Ang-2 in gastric cancer cells. (F) Ang-2 protein levels determined by western blot image analysis. Results are presented as the mean \pm standard error of the mean of three independent experiments. ${ }^{* * *} \mathrm{P}<0.001$. miR, miRNA; Ang-2, angiopoietin-2.

the role of miR-218 in gastric cancer invasion, the basal invasive ability of three gastric cell lines was assessed using the Transwell migration assay. This determined that NCI-87 had a significantly higher invasive ability compared with MGC80-3 and HGC-27 (P<0.001; Fig. 1A). Then, expression levels of miR-218 and Ang-2 in each cell line was measured using RT-qPCR. This identified that NCI-87 had a significantly lower level of miR-218 expression compared with MGC80-3 and HGC-27 (P<0.001; Fig. 1B). Notably, Ang-2 mRNA (Fig. 1C) and protein levels (Fig. 1D and E) were significantly increased in NCI-87 cells compared with MGC80-3 and HGC-27 cells $(\mathrm{P}<0.001)$. These results indicate that miR-218 and Ang-2 serve a role in gastric cancer invasion.
miR-218 overexpression decreases Ang-2 expression, and inhibits the proliferation and migration of gastric cancer cells in vitro. To explore the role of miR-218 in gastric cancer, scrambled miRNA or pre-miR-218 was transfected into NCI-87 and HGC-27 gastric cancer cell lines, which then underwent in vitro proliferation and invasion analyses. Overexpression of miR-218 was found to reduce gastric cancer cell invasion (Fig. 2A) and proliferation (Fig. 2B) in vitro. In addition, in NCI-87 and HGC-27 cells significantly transiently overexpressing miR-218 ( $\mathrm{P}<0.001$ vs. Untransfected; Fig. 2C), Ang-2 protein expression was found to be significantly downregulated compared with the control groups $(\mathrm{P}<0.001)$ via ELISA (Fig. 2D) and western blot analysis (Fig. 2E and F). This suggests that miR-218 regulates the proliferation and 
A
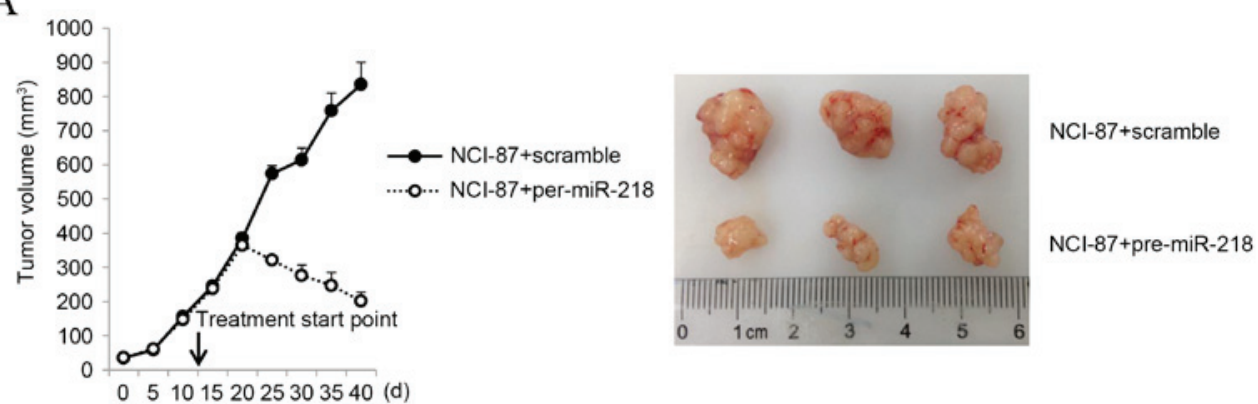

$\mathrm{B}$

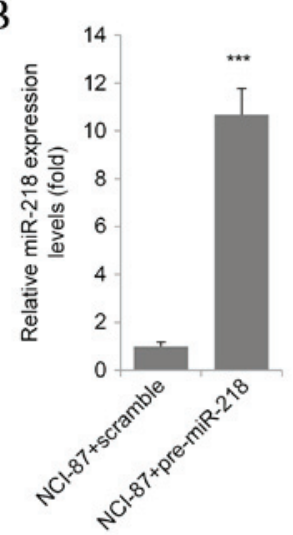

C

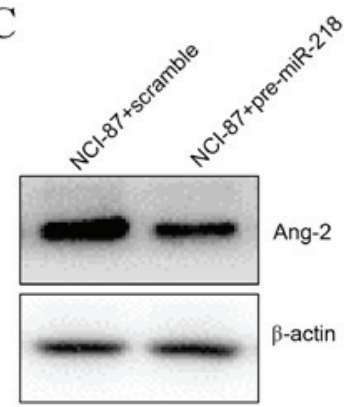

D

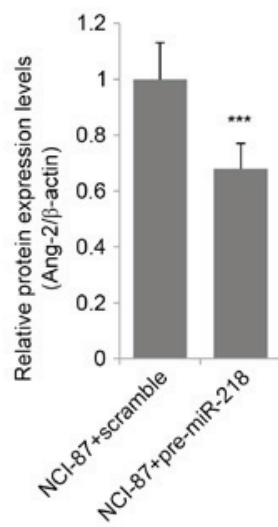

E
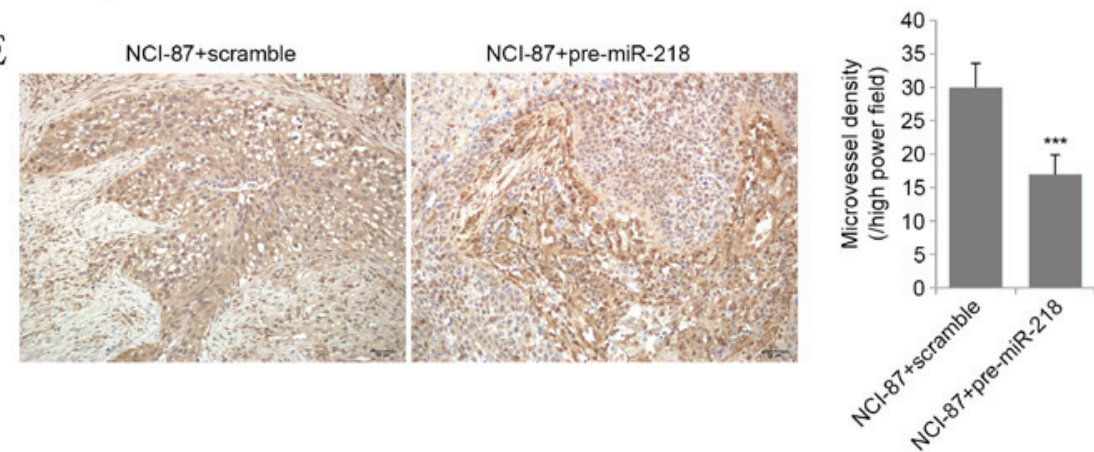

Figure 3. miR-218 inhibits the tumor proliferation and angiogenesis in vivo, concomitantly with decreased expression of Ang-2. (A) Tumor growth curve of nude mice xenografted with NCI- 87 cells, followed by intratumoral injection of pre-miR-218 or scrambled miRNA, and representative images of the tumors of 3 mice from each group at the end of the experiment. (B) Reverse transcription-quantitative polymerase chain reaction analysis of miR-218 expression levels in tumor tissues. (C) Western blot of the protein expression levels of Ang-2 in tumor tissues. (D) Ang-2 protein levels determined by western blot image analysis. (E) Immunohistochemical staining for tumor vascularization and a graphical representation of the results. Results are presented as the mean \pm standard error of the mean of three independent experiments. Scale bar, $50 \mu \mathrm{m}$. ${ }^{* * *} \mathrm{P}<0.001$. miR, miRNA; Ang-2, angiopoietin-2.

migration of gastric cancer cells through regulation of Ang-2 expression.

miR-218 overexpression reduces gastric cancer volume and vascularization in vivo. To determine if miR-218 overexpression decreased the malignancy of gastric cancer cells in vivo, NCI-87 tumor volume in nude mice was assessed prior to and following treatment with pre-miR-218. The results revealed that pre-miR-218 administration to nude mice reduced tumor volume, whereas tumors of the group treated with scrambled miRNA continued to grow (Fig. 3A). RT-qPCR validated that tumor xenografts delivered pre-miR-218 showed significantly higher miR-218 expression compared with the control (Fig. 3B). In addition, pre-miR-218 treatment decreased tumor expression of Ang-2 mRNA ( $\mathrm{P}<0.01$ vs. Control; Fig. 3C) and protein (Fig. 3D).
Tumor vascularization was assessed by Factor VIII, CD31 and Ki-67 immunostaining. Pre-miR-218 treatment significantly decreased tumor MVD compared with the control group $(\mathrm{P}<0.01$; Fig. 3E). These results suggest that miR-218 overexpression inhibits in vivo gastric cancer growth and angiogenesis.

\section{Discussion}

Recently, miRNAs have been reported to promote or suppress tumor proliferation and migration (16-18). The present study demonstrated that endogenous miR-218 expression was significantly decreased in more invasive gastric cancer cell lines. In addition, the results suggested that miR-218 may serve a role in gastric cancer invasion through regulating the expression of Ang-2. Induction of miR-218 overexpression, through the 
transfection of pre-miR-218, caused downregulation of Ang-2 in NCI-87 and HGC-27 gastric cancer cell lines. This was accompanied by a reduction in tumor cell proliferation and migration, suggesting that miR-218 regulates these activities through regulation of Ang-2 expression.

miR-218 was long regarded as a tumor suppressor, for example, miR-218 was reported to inhibit the invasion and metastasis of gastric (19), nasopharyngeal (20), oral (21) and bladder (22) cancer cells. In addition, a previous study determined that miR-218 could inhibit cell cycle progression and promote apoptosis in colorectal cancer (23). In contrast, few reports have demonstrated that miR-218 is able to mediate the expression of Ang-2 to inhibit the angiogenesis in gastric cancer cells. The findings of the current study provide more adequate evidence suggesting that miR-218 functions as a tumor suppressor through regulating the expression of Ang-2.

Notably, the present study identified significantly lower miR-218 and higher Ang-2 expression levels in the more aggressive gastric cancer cell line NCI-87 compared with MGC80-3 and HGC-27. In addition, the results implicated Ang-2 in gastric tumor development through its role in angiogenesis (24). As a primary regulator of angiogenesis, Ang-2 binds to the receptor Tie2 and inhibits Ang-1/Tie2 signaling (25). A previous study found that overexpression of Ang-2 in colon cancer cells was associated with a marked increase in tumor growth rate, vessel count and proliferation (26). In addition, another study found inhibition of Ang-2 slowed tumor growth by inhibiting angiogenesis (27), suggesting that targeting this protein may be a strategy for the treatment of gastric cancer.

Based on the in vitro results, suggesting that miR-218 and Ang-2 are involved in gastric cancer development, the in vivo effects of exogenous miR-218 expression of miR-218 were explored. Intratumoral delivery of pre-miR-218 to nude mice reduced NCI-87 tumor volume and vascularization. This reduction in tumorigencity was accompanied with a reduction in the expression of Ang-2 in tumor xenografts.

In conclusion, the results of the current study indicate that the miR-218/Ang-2 axis could be a therapeutic target for gastric cancer therapy. However, the extent to which miR-218/Ang-2 signaling promotes the progression of gastric cancer needs to be further investigated.

\section{References}

1. Ferlay J, Shin HR, Bray F, Forman D, Mathers C and Parkin DM: Estimates of worldwide burden of cancer in 2008: GLOBOCAN 2008. Int J Cancer 127: 2893-2917, 2010.

2. Sasako M, Sakuramoto S, Katai H, Kinoshita T, Furukawa H, Yamaguchi T, Nashimoto A, Fujii M, Nakajima T and Ohashi Y: Five-year outcomes of a randomized phase III trial comparing adjuvant chemotherapy with S-1 versus surgery alone in stage II or III gastric cancer. J Clin Oncol 29: 4387-4393, 2011.

3. Bray F, Jemal A, Grey N, Ferlay J and Forman D: Global cancer transitions according to the Human Development Index (2008-2030): A population-based study. Lancet Oncol 13: 790-801, 2012.

4. Tu SP, Jiang XH, Lin MC, Cui JT, Yang Y, Lum CT, Zou B, Zhu YB, Jiang SH, Wong WM, et al: Suppression of survivin expression inhibits in vivo tumorigenicity and angiogenesis in gastric cancer. Cancer Res 63: 7724-7732, 2003.

5. Blank S, Deck C, Dreikhausen L, Weichert W, Giese N, Falk C, Schmidt T and Ott K: Angiogenic and growth factors in gastric cancer. J Surg Res 194: 420-429, 2015.

6. Gerald D, Chintharlapalli S, Augustin HG and Benjamin LE: Angiopoietin-2: An attractive target for improved antiangiogenic tumor therapy. Cancer Res 73: 1649-1657, 2013.
7. Mitsuhashi N, Shimizu H, Ohtsuka M, Wakabayashi Y, Ito H, Kimura F, Yoshidome H, Kato A, Nukui Y and Miyazaki M: Angiopoietins and Tie-2 expression in angiogenesis and proliferation of human hepatocellular carcinoma. Hepatology 37: 1105-1113, 2003.

8. Maisonpierre PC, Suri C, Jones PF, Bartunkova S, Wiegand SJ, Radziejewski C, Compton D, McClain J, Aldrich TH, Papadopoulos N, et al: Angiopoietin-2, a natural antagonist for Tie2 that disrupts in vivo angiogenesis. Science 277: 55-60, 1997.

9. Stratmann A, Risau W and Plate KH: Cell type-specific expression of angiopoietin-1 and angiopoietin-2 suggests a role in glioblastoma angiogenesis. Am J Pathol 153: 1459-1466, 1998.

10. Urbich C, Kuehbacher A and Dimmeler S: Role of microRNAs in vascular diseases, inflammation, and angiogenesis. Cardiovasc Res 79: 581-588, 2008.

11. Fish JE and Srivastava D: MicroRNAs: Opening a new vein in angiogenesis research. Sci Signal 2: pe1, 2009.

12. Weng C, Dong H, Chen G, Zhai Y, Bai R, Hu H, Lu L and Xu Z: miR-409-3p inhibits HT1080 cell proliferation, vascularization and metastasis by targeting angiogenin. Cancer Lett 323: 171-179, 2012.

13. Bai J, Zhang J, Wu J, Shen L, Zeng J, Ding J, Wu Y, Gong Z, $\mathrm{Li} \mathrm{A}, \mathrm{Xu} \mathrm{S}$, et al: JWA regulates melanoma metastasis by integrin alphaVbeta3 signaling. Oncogene 29: 1227-1237, 2010.

14. Livak KJ and Schmittgen TD: Analysis of relative gene expression data using real-time quantitative PCR and the 2(-Delta Delta C(T)) Method. Methods 25: 402-408, 2001.

15. Tomayko MM and Reynolds CP: Determination of subcutaneous tumor size in athymic (nude) mice. Cancer Chemother Pharmacol 24: 148-154, 1989

16. Cai LM, Lyu XM, Luo WR, Cui XF, Ye YF, Yuan CC, Peng QX, Wu DH, Liu TF, Wang E, et al: EBV-miR-BART7-3p promotes the EMT and metastasis of nasopharyngeal carcinoma cells by suppressing the tumor suppressor PTEN. Oncogene 34: 2156-2166, 2015.

17. Kim HS, Lee KS, Bae HJ, Eun JW, Shen Q, Park SJ, Shin WC, Yang HD, Park M, Park WS, et al: MicroRNA-31 functions as a tumor suppressor by regulating cell cycle and epithelial-mesenchymal transition regulatory proteins in liver cancer. Oncotarget 6: 8089-8102, 2015.

18. Chen D, Guo W, Qiu Z, Wang Q, Li Y, Liang L, Liu L, Huang S, Zhao Y and He X: MicroRNA-30d-5p inhibits tumour cell proliferation and motility by directly targeting CCNE2 in non-small cell lung cancer. Cancer Lett 362: 208-217, 2015.

19. Tie J, Pan Y, Zhao L, Wu K, Liu J, Sun S, Guo X, Wang B, Gang Y,Zhang Y, et al: MiR-218 inhibits invasion and metastasis of gastric cancer by targeting the Robol receptor. PLoS Genet 6: e1000879, 2010.

20. Alajez NM, Lenarduzzi M, Ito E, Hui AB, Shi W, Bruce J, Yue S, Huang $\mathrm{SH}, \mathrm{Xu} \mathrm{W}$, Waldron J, et al: MiR-218 suppresses nasopharyngeal cancer progression through downregulation of survivin and the SLIT2-ROBO1 pathway. Cancer Res 71: 2381-2391, 2011.

21. Uesugi A, Kozaki K, Tsuruta T, Furuta M, Morita K, Imoto I, Omura $\mathrm{K}$ and Inazawa J: The tumor suppressive microRNA miR-218 targets the mTOR component Rictor and inhibits AKT phosphorylation in oral cancer. Cancer Res 71: 5765-5778, 2011.

22. Tatarano S, Chiyomaru T, Kawakami K, Enokida H, Yoshino H, Hidaka H, Yamasaki T, Kawahara K, Nishiyama K, Seki N and Nakagawa M: miR-218 on the genomic loss region of chromosome 4p15. 31 functions as a tumor suppressor in bladder cancer. Int J Oncol 39: 13-21, 2011.

23. Ng EK, Chong WW, Jin H, Lam EK, Shin VY, Yu J, Poon TC, Ng SS and Sung JJ: Differential expression of microRNAs in plasma of patients with colorectal cancer: A potential marker for colorectal cancer screening. Gut 58: 1375-1381, 2009.

24. Etoh T, Inoue H, Tanaka S, Barnard GF, Kitano S and Mori M: Angiopoietin-2 is related to tumor angiogenesis in gastric carcinoma: Possible in vivo regulation via induction of proteases. Cancer Res 61: 2145-2153, 2001.

25. Felcht M, Luck R, Schering A, Seidel P, Srivastava K, Hu J, Bartol A, Kienast Y, Vettel C, Loos EK, et al: Angiopoietin-2 differentially regulates angiogenesis through TIE2 and integrin signaling. J Clin Invest 122: 1991-2005, 2012.

26. Ahmad SA, Liu W, Jung YD, Fan F, Wilson M, Reinmuth N, Shaheen RM, Bucana CD and Ellis LM: The effects of angiopoietin-1 and-2 on tumor growth and angiogenesis in human colon cancer. Cancer Res 61: 1255-1259, 2001.

27. Hashizume H, Falcón BL, Kuroda T, Baluk P, Coxon A, Yu D, Bready JV, Oliner JD and McDonald DM: Complementary actions of inhibitors of angiopoietin-2 and VEGF on tumor angiogenesis and growth. Cancer Res 70: 2213-2223, 2010. 